\title{
Age constraints and fine tuning in variable-mass particle models
}

\author{
Urbano França* and Rogerio Rosenfeld ${ }^{\dagger}$ \\ Instituto de Física Teórica-UNESP, Rua Pamplona, 145, 01405-900, São Paulo, SP, Brazil
}

(Received 13 October 2003; published 25 March 2004)

\begin{abstract}
VAMP (variable-mass particle) scenarios, in which the mass of the cold dark matter particles is a function of the scalar field responsible for the present acceleration of the Universe, have been proposed as a solution to the cosmic coincidence problem, since in the attractor regime both dark energy and dark matter scale in the same way. We find that only a narrow region in parameter space leads to models with viable values for the Hubble constant and dark energy density today. In the allowed region, the dark energy density starts to dominate around the present epoch and consequently such models cannot solve the coincidence problem. We show that the age of the Universe in this scenario is considerably higher than the age for noncoupled dark energy models, and conclude that more precise independent measurements of the age of the Universe would be useful in distinguishing between coupled and noncoupled dark energy models.
\end{abstract}

DOI: 10.1103/PhysRevD.69.063517

PACS number(s): 98.80.Cq

\section{INTRODUCTION}

The Wilkinson Microwave Anisotropy Probe (WMAP) satellite [1] has confirmed that the Universe is very nearly flat, and that there is some form of dark energy (DE) that is the current dominant energy component, accounting for approximately $70 \%$ of the critical density. DE is smoothly distributed throughout the Universe and is causing its present acceleration [2,3]. It is generally modeled using a scalar field, the so-called quintessence models, either slowly rolling towards the minimum of the potential or already trapped in this minimum $[2,4-6]$. WMAP has also given new observational evidence that nonbaryonic cold dark matter (DM), a fluid with vanishing pressure, contributes around $25 \%$ to the total energy density of the Universe, in agreement with a set of cosmological observations [3,7].

An intriguing possibility is that DM particles could interact with the DE field, resulting in a time-dependent mass. In this scenario, dubbed VAMPs (variable-mass particles) [8], the mass of the DM particles evolves according to some function of the dark energy field $\phi$, such as, for example, a linear function of the field [8-11] with an inverse power law dark energy potential or an exponential function [12-16] with an exponential dark energy potential. In this work we are particularly interested in the exponential case, since it presents a tracker solution; ${ }^{1}$ that is, there is a stable attractor regime where the effective equation of state of $\mathrm{DE}$ mimics the effective equation of state of DM $[12,16]$.

This type of solution also appears when the DE field with an exponential potential is not coupled to the other fluids $[6,17]$. In fact, Liddle and Scherrer showed that for a noncoupled DE, the exponential potential is the only one that presents stable tracker solutions [18]. In this case, however, it is not able to explain the current acceleration of the Uni-

\footnotetext{
*Electronic address: urbano@ift.unesp.br

†Electronic address: rosenfel@ift.unesp.br

${ }^{1}$ We adopt the nomenclature "scaling" solution to denote solutions where $\rho_{\phi} \propto a^{-m}$ and $\rho_{m} \propto a^{-n}$, whereas a "tracker" solution has $m=n$.
}

verse and other observational constraints, unless we assume that the field has not yet reached the fixed point regime [19].

The tracker behavior is interesting because once the attractor is reached, the ratio between DM energy density $\rho_{\chi}$ and DE energy density $\rho_{\phi}$ remains constant afterwards. ${ }^{2}$ This behavior could solve the "cosmic coincidence problem," that is: why are the DE and DM energy densities similar today?

In this article, we want to study the extent of fine tuning required in this class of models given the observational constraints on the Hubble constant $H_{0}$ and the dark energy density today $\Omega_{\phi 0}$. We also compute the resulting age of the Universe for the allowed models. In Sec. II we present the main equations of the VAMP scenario and the constraints that have already been discussed elsewhere. In Sec. III we show that based on very robust constraints such models are not able to solve the cosmic coincidence problem. Section IV discusses the constraints imposed by the age of the Universe in these models, showing that better model-independent measurements of a lower limit would provide a possible way to distinguish between the noncoupled and coupled dark energy models. In Sec. V we present our conclusions.

\section{THE IDEA OF EXPONENTIAL VAMPS}

In the exponential VAMP model, the potential of the DE scalar field $\phi$ is given by

$$
V(\phi)=V_{0} e^{\beta \phi / m_{p}},
$$

where $V_{0}$ and $\beta$ are positive constants and $m_{p}=M_{p} / \sqrt{8 \pi}$ $=2.436 \times 10^{18} \mathrm{GeV}$ is the reduced Planck mass in natural units, $\hbar=c=1$. Dark matter is modeled by a scalar particle $\chi$ of mass

$$
M_{\chi}=M_{\chi 0} e^{-\lambda\left(\phi-\phi_{0}\right) / m_{p}},
$$

\footnotetext{
${ }^{2}$ This behavior does not occur in the situation in which the mass varies linearly with the field, although one can let the ratio between $\rho_{\chi}$ and $\rho_{\phi}$ remain constant for a long time, until the DE finally comes to dominate completely [11].
} 
where $M_{\chi 0}$ is the current mass of the dark matter particle (hereafter the index 0 denotes the present epoch, except for the potential constant $V_{0}$ ) and $\lambda$ is a positive constant. Since dark matter must be stable, its number density $n_{\chi}$ obeys the equation

$$
\dot{n}_{\chi}+3 H n_{\chi}=0 \text {, }
$$

where the dot denotes a derivative with respect to time, $H$ $=\dot{a} / a$, and $a(t)$ is the scale factor. Assuming that the dark matter is nonrelativistic, its energy density is given by $\rho_{\chi}$ $=M_{\chi} n_{\chi}$, and it follows that

$$
\dot{\rho}_{\chi}+3 H \rho_{\chi}=-\frac{\lambda \dot{\phi}}{m_{p}} \rho_{\chi} .
$$

Since the total energy-momentum tensor has to be conserved, the fluid equation for dark energy is given by

$$
\dot{\rho}_{\phi}+3 H \rho_{\phi}\left(1+\omega_{\phi}\right)=\frac{\lambda \dot{\phi}}{m_{p}} \rho_{\chi},
$$

where $\omega_{\phi}=p_{\phi} / \rho_{\phi}=\left(\frac{1}{2} \dot{\phi}^{2}-V\right) /\left(\frac{1}{2} \dot{\phi}^{2}+V\right)$ is the usual equation of state parameter for an homogeneous scalar field.

These equations can also be written in the form

$$
\begin{aligned}
& \dot{\rho}_{\chi}+3 H \rho_{\chi}\left(1+\omega_{\chi}^{(e)}\right)=0, \\
& \dot{\rho}_{\phi}+3 H \rho_{\phi}\left(1+\omega_{\phi}^{(e)}\right)=0,
\end{aligned}
$$

where

$$
\begin{aligned}
& \omega_{\chi}^{(e)}=\frac{\lambda \dot{\phi}}{3 H m_{p}}=\frac{\lambda \phi^{\prime}}{3 m_{p}}, \\
& \omega_{\phi}^{(e)}=\omega_{\phi}-\frac{\lambda \dot{\phi}}{3 H m_{p}} \frac{\rho_{\chi}}{\rho_{\phi}}=\omega_{\phi}-\frac{\lambda \phi^{\prime}}{3 m_{p}} \frac{\rho_{\chi}}{\rho_{\phi}}
\end{aligned}
$$

are the effective equation of state parameters for dark matter and dark energy, respectively. Primes denote derivatives with respect to $u=\ln (a)=-\ln (1+z)$, where $z$ is the redshift, and $a_{0}=1$. From the above equations one can also obtain the $\mathrm{DE}$ equation of motion,

$$
\ddot{\phi}+3 H \dot{\phi}=\frac{\lambda \rho_{\chi}}{m_{p}}-\frac{\beta V}{m_{p}} .
$$

The Friedmann equation for a universe with DE, DM, baryons, and radiation is given by

$$
H^{2}=\frac{1}{3 m_{p}^{2}}\left[\rho_{b}+\rho_{r}+\rho_{\chi}+\frac{1}{2} \dot{\phi}^{2}+V(\phi)\right],
$$

where we have assumed that the universe is flat,

$$
\Omega_{0} \equiv \frac{\rho_{0}}{\rho_{c 0}}=\Omega_{\phi 0}+\Omega_{\chi 0}+\Omega_{b 0}+\Omega_{r 0}=1
$$

where $\rho_{c 0}=3 m_{p}^{2} H_{0}^{2}=8.1 h^{2} \times 10^{-47} \mathrm{GeV}^{4}$ is the present critical density and the Hubble parameter is $H_{0}$ $=100 \mathrm{~km} \mathrm{~s}^{-1} \mathrm{Mpc}^{-1} \cdot \rho_{b}$ and $\rho_{r}$ denote the energy densities of baryons and radiation, respectively, which for $i=r, b$ satisfy the equations $\dot{\rho}_{i}+3 H \rho_{i}\left(1+\omega_{i}\right)=0$, where $\omega_{b}=0$ and $\omega_{r}=1 / 3$. It is useful to express $V_{0}$ in units of

$$
\tilde{\rho}_{c}=4.2 \times 10^{-47} \mathrm{GeV}^{4},
$$

the critical density value for $h=0.72$. Observational limits on the Hubble parameter give $h=0.72 \pm 0.08$ [20], which we adopt in what follows.

The Friedmann and DE motion equations become, in terms of $u$,

$$
\begin{gathered}
H^{2} \phi^{\prime \prime}+\frac{1}{3 m_{p}^{2}}\left[\frac{3}{2}\left(\rho_{b}+\rho_{\chi}\right)+\rho_{r}+3 V\right] \phi^{\prime}=\frac{\lambda \rho_{\chi}}{m_{p}}-\frac{\beta V}{m_{p}}, \\
H^{2}=\frac{\left(1 / 3 m_{p}^{2}\right)\left(\rho_{\chi}+\rho_{b}+\rho_{r}+V\right)}{1-\left(1 / 6 m_{p}^{2}\right) \phi^{\prime 2}} .
\end{gathered}
$$

Using the fact that the right-hand side of Eq. (14) is the derivative with respect to the field $\phi$ of an effective potential [11],

$$
V_{e f f}(\phi)=V(\phi)+\rho_{\chi}(\phi),
$$

one can show that there is a fixed point value for the field, given by $d V_{e f f}(\phi) / d \phi=0$ :

$$
\frac{\phi}{m_{p}}=-\frac{3}{(\lambda+\beta)} u+\frac{1}{(\lambda+\beta)} \ln \left(\frac{\beta V_{0}}{\lambda \rho_{\chi 0} e^{\lambda \phi_{0} / m_{p}}}\right) .
$$

At the present epoch the energy density of the Universe is divided essentially between dark energy and dark matter. In this limit, using the above solution, one obtains

$$
\begin{aligned}
& \Omega_{\phi}=1-\Omega_{\chi}=\frac{3}{(\lambda+\beta)^{2}}+\frac{\lambda}{\lambda+\beta}, \\
& \omega_{\chi}^{(e)}=\omega_{\phi}^{(e)}=-\frac{\lambda}{\lambda+\beta},
\end{aligned}
$$

which is a stable attractor for $\beta>-\lambda / 2+\left(\sqrt{\lambda^{2}+12}\right) / 2$ $[12,16]$. The equality between $\omega_{\chi}$ and $\omega_{\phi}$ in the attractor regime comes from the tracker behavior of the exponential potential $[12,16,17]$ in this regime.

The density parameters for the components of the Universe and the effective equations of state for the DE and DM for a typical solution are shown in Fig. 1. Notice that the transition to the tracker behavior in this example is currently occurring.

Let us assume observational upper and lower limits to the DE equation of state parameters $\omega_{o b s}^{l} \leqslant \omega_{\phi 0}^{(e)} \leqslant \omega_{o b s}^{u}$ from SNIa, for instance. Then, from Eq. (19) it follows a constraint on the parameter space $(\lambda, \beta)$ given by 

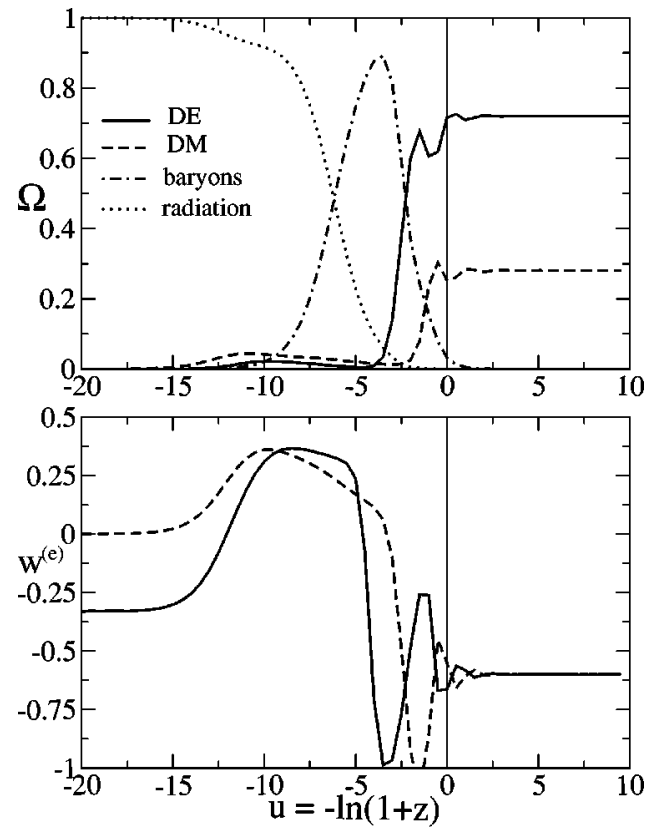

FIG. 1. Top panel: Density parameters of the components of the Universe as a function of $u=-\ln (1+z)$ for $\lambda=3, \beta=2$, and $V_{0}$ $=0.1 \tilde{\rho}_{c}$. After a transient period of baryonic matter domination (dot-dashed line), DE comes to dominate and the ratio between the DE (solid line) and DM (dashed line) energy densities remains constant. Bottom panel: Effective equations of state for DE (solid line) and DM (dashed line) for the same parameters used in top panel. In the tracker regime both equations of state are negative.

$$
\beta \leqslant(\geqslant) \frac{1-\left|\omega_{o b s}^{u(l)}\right|}{\left|\omega_{o b s}^{u(l)}\right|} \lambda,
$$

which is valid if the field has already reached the attractor solution.

Analogously, in the attractor solution, the density parameter $\Omega_{\phi 0}$ can also constrain the parameter space, since Eq. (18) implies

$$
\begin{aligned}
\beta \geqslant(\leqslant) \frac{\left(1-2 \Omega_{o b s}^{(D E) u(l)}\right)}{2 \Omega_{o b s}^{(D E) u(l)}} \lambda & \\
+ & \frac{\sqrt{\lambda^{2}\left[\left(2 \Omega_{o b s}^{(D E) u(l)}-1\right)^{2}-4\left(\Omega_{o b s}^{(D E) u(l)}-1\right)\right]+12}}{2 \Omega_{o b s}^{(D E) u(l)}},
\end{aligned}
$$

where $\Omega_{o b s}^{(D E) u(l)}$ is the upper (lower) limit to the current value of DE density parameter.

Amendola [14] has studied in detail the observational constraints imposed in this scenario by the dark energy equation of state (obtained from luminosity distance consistent with high $z$ supernovae) and density parameters when the attractor regime has already been reached. He obtained $-0.8 \leqslant \omega_{\phi 0}^{(e)} \leqslant-0.4$ at $95 \%$ confidence limit (C.L.). Figure 2 shows the allowed region in the $(\lambda, \beta)$ parameter space using

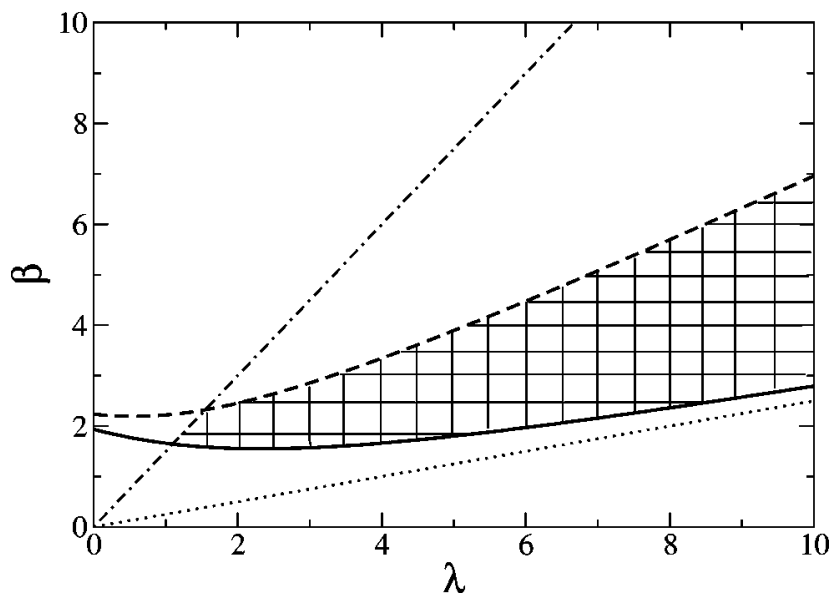

FIG. 2. Constraints on parameter space $(\lambda, \beta)$ given by DE equation of state and DE density parameter. The upper (lower) limit on equation of state allows the regions under (above) the dot-dashed (dotted) line for $\omega_{o b s}=-0.4(-0.8)$. On the other hand, the upper (lower) limit on the DE density parameter, $\Omega_{o b s}^{(D E)}=0.8(0.6)$, requires the parameters to be above (under) the solid (dashed) line. The hatched region is allowed by both constraints.

this result together with a conservative bound $0.6 \leqslant \Omega_{\phi 0}$ $\leqslant 0.8$ on the DE density parameter.

\section{FINE TUNING}

Tracker solutions of noncoupled exponential quintessence models in which the dark energy equation of state tracks that of the background are not able to accelerate the Universe today. Exponential VAMPs solve this problem because the background acquires a negative equation of state. However, it is necessary to verify whether fine tuning of the model is necessary to explain the observations today.

In order to quantify the amount of fine tuning required, we have solved numerically the coupled equations of motion for the scalar field, varying $V_{0}, \lambda$, and $\beta$ in the region $\lambda$ $=[0.01,20], \beta=[0.01,20]$, and $V_{0}=\left[0.01 \tilde{\rho}_{c}, 1.5 \tilde{\rho}_{c}\right]$, with step sizes $\Delta \lambda=\Delta \beta=0.25$ and $\Delta V_{0}=0.05 \tilde{\rho}_{c}$, generating about $1.9 \times 10^{5}$ models, from which we have selected those that agree with observed cosmological parameters.

Figure 3 shows the normalized number of models that satisfy some observational constraints as function of $V_{0}$, given in units of $\tilde{\rho}_{c}$. The dashed line shows the normalized number of models that satisfy the Hubble parameter constraint, $h=0.72 \pm 0.08$ [20]. Notice that in order for this constraint to be satisfied it requires $V_{0}$ to be of the order of $\tilde{\rho}_{c}$, the critical density today. The dot-dashed line is obtained requiring both the Hubble parameter constraint and the equation of state constraint, $-0.8 \leqslant \omega_{\phi 0}^{(e)} \leqslant-0.4$ at $95 \%$ C.L. [14]. The dotted line corresponds to the case when the Hubble parameter constraint and parameter density constraint, 0.6 $\leqslant \Omega_{\phi 0} \leqslant 0.8$, are taken into account, and the solid line when these three parameters are constrained simultaneously. One can see that the narrow allowed range of $V_{0}$ $=\left[0.25 \tilde{\rho}_{c}, 0.45 \tilde{\rho}_{c}\right], 68 \%$ C.L. $\left(V_{0}=\left[0.15 \tilde{\rho}_{c}, 0.55 \tilde{\rho}_{c}\right], 95 \%\right.$ C.L.), is essentially determined by the constraints given by $h$ and $\Omega_{\phi 0}$, which are very conservative and robust. Hence, fine tuning in this class of models seems unavoidable.

What does this fine tuning in $V_{0}$ mean? $V_{0}$ is the main 


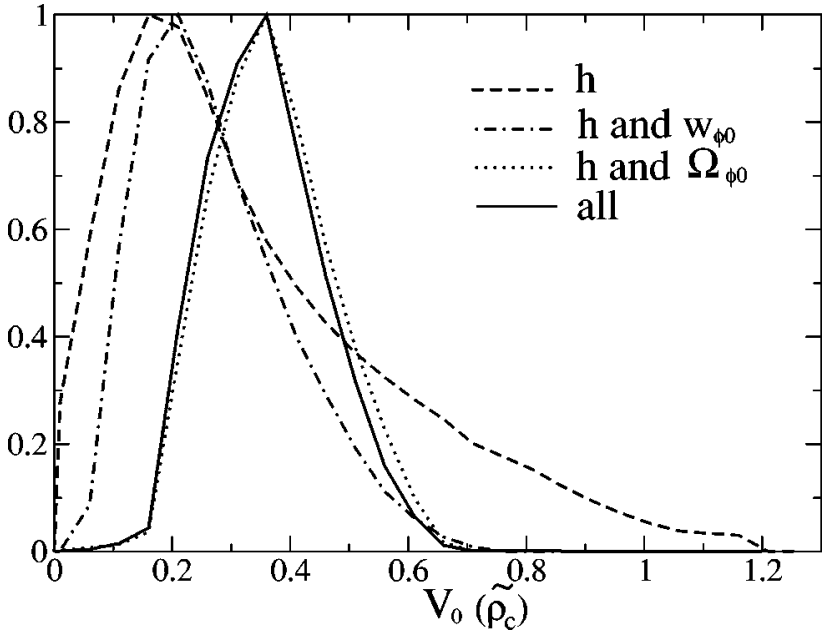

FIG. 3. Normalized number of models that satisfy the observational constraints discussed in the text.

parameter that determines the epoch in which the field reaches the attractor regime. As can be seen in Fig. 1, for the allowed values of $V_{0}$, the Universe is entering the attractor regime around the present epoch. This implies that the equation of state and the parameter density are still varying today, although their current values are very similar to the values at the fixed point.

However, if one varies $V_{0}$ a similar behavior of $\Omega_{D E}$ and $\Omega_{D M}$ sets in earlier or later, as can be seen in Fig. 4. However, unrealistic values of the Hubble parameter result in this case. For instance, for $V_{0}=10^{3} \tilde{\rho}_{c}$, where the fixed point is reached earlier, we obtain $h \approx 10$. In this sense, the cosmic coincidence problem is not solved in these models, since one has to choose the overall scale of the potential in order to obtain realistic cosmological solutions, very much like noncoupled dark energy models.

\section{AGE OF THE UNIVERSE}

The potential to use the age of the Universe as a constraint to dark energy models has been discussed in several
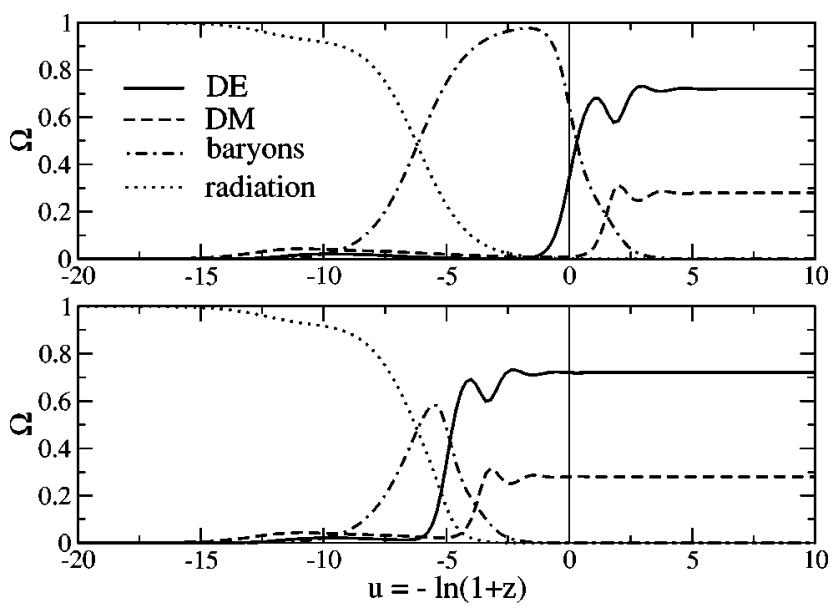

FIG. 4. Density parameters of the components of the Universe as function of $u=\ln (1+z)$ for $\lambda=3, \beta=2$. In the top panel $V_{0}$ $=10^{-4} \tilde{\boldsymbol{\rho}}_{c}$, and in the bottom panel $V_{0}=10^{3} \tilde{\boldsymbol{\rho}}_{c}$. recent papers [21-24]. The WMAP collaboration [1] has given a stringent value for the age of the Universe, $t_{0}$ $=13.7 \pm 0.2 \mathrm{Gyr}$ at $68 \%$ C.L. However, this result is model dependent and obtained from direct integration of the Friedmann equation for the running spectral index cold dark matter with a cosmological constant $(\Lambda \mathrm{CDM})$ model. Observations also indicate a lower limit for the age of the oldest globular clusters (and consequently for the age of the Universe) of $t_{0}=10.4$ Gyr at $95 \%$ C.L. [21].

Using the limits discussed above and the position of the first Doppler peak in the WMAP experiment it is possible to put an upper limit on the equation of state (assumed constant) of the dark energy, $\omega_{\phi}<-0.67$ at $90 \%$ C.L. [22]. However, current upper limits on the age of the Universe alone cannot constrain the lower value of the equation of state, since for large values of the age $\left(t_{0} \gtrsim 18 \mathrm{Gyr}\right)$ the cosmology is essentially independent of the value of the equation of state [23], unless we assume that reionization took place too early in the Universe history (as indicated by WMAP data), and consequently the upper limit for the age of the Universe is close to the upper limits coming from globular clusters, $t_{0} \approx 16 \mathrm{Gyr}$, as in the case discussed in Ref. [22].

A model independent approach to estimating the age of the Universe has been performed by means of a parametrization for the equation of state of dark energy that allows it to vary [24]. Using cosmic microwave background (CMB) and supernova data the age of the Universe was found to be $t_{0}=13.8 \pm 0.3 \mathrm{Gyr}$ at $68 \%$ C.L. Even this model independent approach, however, is not able to parametrize the VAMP scenario, since in this case the effective equation of state of the CDM is also variable, and the strong limits in the age of the Universe obtained by these CMB analyses cannot be used to constrain the models discussed here. For this reason, we have used only the conservative limits obtained from globular clusters to study the potential of the age of the Universe to constrain this scenario. Therefore we adopt $10.7<t_{0}$ $<16.3 \mathrm{Gyr}$ at $95 \%$ C.L. [21]. We have added to the age of globular clusters $0.3 \mathrm{Gyr}$, since the WMAP data indicate an early reionization of the Universe $[1,22]$. The age of the Universe in the VAMP scenario is given by

$$
\begin{aligned}
t_{0}= & H_{0}^{-1} \int_{0}^{z_{i}}\left\{\Omega_{\phi 0} \exp \left[3 \int_{-u_{i}}^{0}\left[1+\omega_{\phi}^{(e)}(u)\right] d u\right]\right. \\
& +\Omega_{b 0}(1+z)^{3}+\Omega_{r 0}(1+z)^{4} \\
& \left.+\Omega_{\chi 0} \exp \left[3 \int_{-u_{i}}^{0}\left[1+\omega_{\chi}^{(e)}(u)\right] d u\right]\right\}^{-1 / 2} \frac{d z}{(1+z)},
\end{aligned}
$$

where $u_{i}$ is the initial value for $u\left(u_{i}=-30\right.$ for our numerical calculations) and $z_{i}$ its corresponding redshift.

To explore the region of parameter space that is able to satisfy the age constraint we have used the same procedure described in the preceding section, varying $\lambda$ and $\beta$ in the region $[0.01,20]$, with a step size $\Delta \lambda=\Delta \beta=0.05$ and a fixed value of $V_{0}$, generating about $1.6 \times 10^{5}$ models. Models were required to satisfy the constraints on the Hubble parameter and on the age of the Universe. Results are shown in Fig. 5, for three different values of $V_{0}$. 


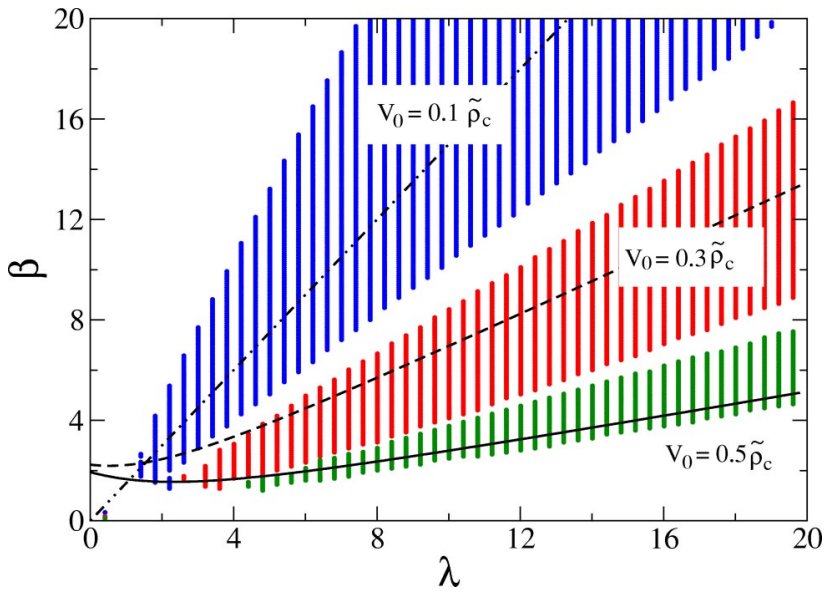

FIG. 5. (Color online) Constraints of parameter space $(\lambda, \beta)$ imposed by the age of the Universe for $V_{0}=0.1 \tilde{\rho}_{c}, V_{0}=0.3 \tilde{\rho}_{c}$, and $V_{0}=0.5 \tilde{\rho}_{c}$. Lines correspond to the same limits from Fig. 2.

The constraints imposed by the age of the Universe are in the same direction of those imposed by the dark energy density and the equation of state, indicating a correlation among them. This can be seen explicitly in Fig. 6. Notice that such a correlation is exactly the opposite that occurs when the equation of state (for noncoupled dark energy) is taken to be constant [22]: higher values of $\omega_{\phi 0}^{(e)}$ imply lower ages of the Universe.

In the constant case, the relation between the age and $\omega$ is direct: for more negative equations of state the contribution of dark energy becomes important later (since $\rho$ scales as $\left.a^{-3(1+\omega)}\right)$. Consequently, for a longer time the Universe is matter dominated, and its age is lower compared with a universe in which the dark energy has a more positive equation of state.

For the VAMP scenario, however, the situation is a little more complicated: models that satisfy the age of the Universe consist of a positive correlation in the parameter space that has a lower slope for larger $V_{0}$ (Fig. 5). For larger $V_{0}$, the Universe reaches the attractor regime earlier, and conse-

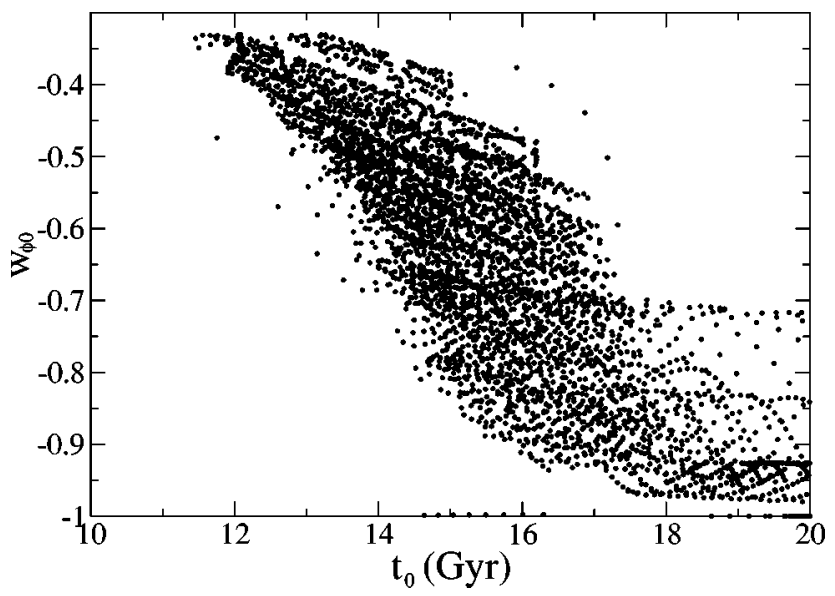

FIG. 6. Age of the Universe versus the present dark energy equation of state for models that satisfy the Hubble parameter constraint.

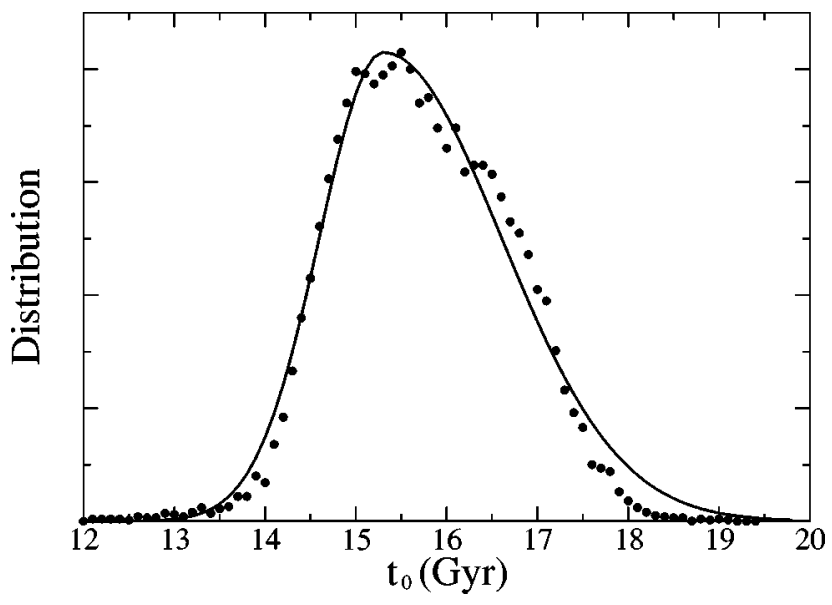

FIG. 7. Distribution of models that satisfy Hubble and DE density parameters constraints as a function of age of the Universe. Fit to the points gives $t_{0}=15.3_{-0.7}^{+1.3} \mathrm{Gyr}$ at $68 \%$ C.L.

quently has a larger value of the age of the Universe (for the same $\beta$ and $H_{0}$ ), since the Universe is accelerating for a longer time. Besides that, for the same $\beta$, larger values of $V_{0}$ imply larger values of $\lambda$ for the models that satisfy the age constraint, and consequently, from Eq. (19), the equation of state tends to -1 . Despite this correlation, due the large degeneracy in the allowed region, it seems to be very difficult to constrain $\omega_{\phi 0}^{(e)}$ based on limits on $t_{0}$ and vice versa.

Figure 7 shows the age of the Universe for the models that satisfy the Hubble parameter and the dark energy density observational constraints. Here we have used step sizes $\Delta \lambda$ $=\Delta \beta=0.2$ and $\Delta V_{0}=0.05 \tilde{\rho}_{c}$ for the region $\lambda=[0.01,20]$, $\beta=[0.01,20], V_{0}=\left[0.1 \tilde{\rho}_{c}, 0.8 \tilde{\rho}_{c}\right]$.

Fitting the distribution of models as function of ages, the age of the Universe in this VAMP scenario was found to be

$$
t_{0}=15.3_{-0.7}^{+1.3} \text { Gyr at } 68 \% \text { C.L., }
$$

which is considerably higher than the age of models of noncoupled dark energy [1,24]. This seems natural, since in these models the CDM also has an effective negative equation of state and accelerates the Universe. Thus, measurements of the age of the Universe could help to distinguish between coupled and noncoupled models. This result is very conservative, since it relies only on the well established limits of the Hubble constant and the dark energy density today.

One can see that lower limits on the age of the Universe are not useful to constrain these models. Upper limits on the age, however, are potentially interesting. As an example, we can speculate what would change if the limits on the age of globular clusters [21,22] were symmetric, $t_{0}=12.5$ $\pm 2.2 \mathrm{Gyr}$, and add to this age $0.3 \mathrm{Gyr}$ for the formation of these objects. In this case, the upper limit on the age of the Universe would be $15 \mathrm{Gyr}$, which would exclude a large part of the models allowed by the present limits. The parameter space for these limits (Fig. 8) is much more constrained than the previous case, practically excluding $V_{0}>0.5 \tilde{\rho}_{c}$. 


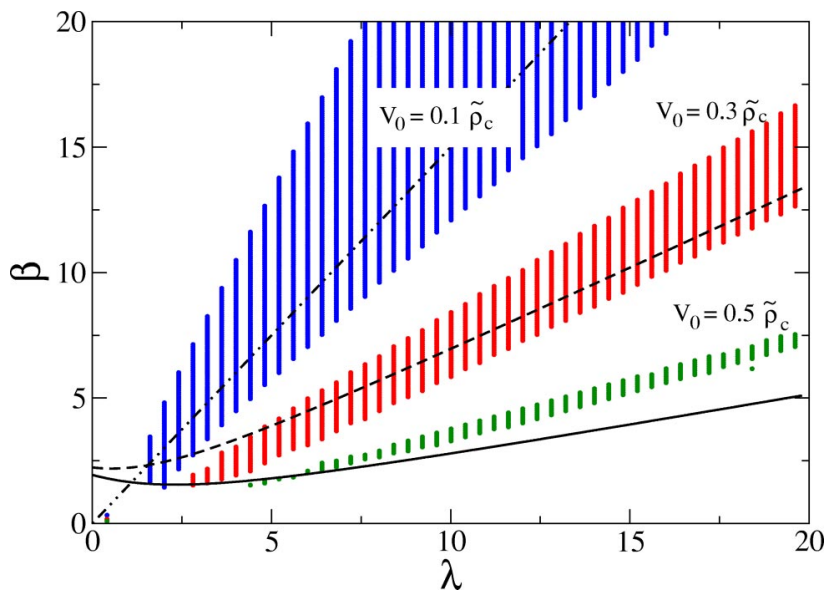

FIG. 8. (Color on line) Constraints of parameter space $(\lambda, \beta)$ imposed by $t_{0}=12.5 \pm 2.2 \mathrm{Gyr}$, and adding $0.3 \mathrm{Gyr}$ as the age of the Universe in the epoch the globular clusters were formed, for $V_{0}=0.1 \tilde{\rho}_{c}, V_{0}=0.3 \tilde{\rho}_{c}$, and $V_{0}=0.5 \tilde{\rho}_{c}$. Lines correspond to the same limits from Fig. 2.

\section{CONCLUSION}

The VAMP scenario is attractive since it could solve the problems of exponential dark energy, giving rise to a solution of the cosmic coincidence problem. However, in order to obtain solutions that can provide realistic cosmological parameters, the constant $V_{0}$ has to be extremely fine tuned in the range $V_{0}=\left[0.25 \tilde{\rho}_{c}, 0.45 \tilde{\rho}_{c}\right]$ at $68 \%$ C.L. This implies that the attractor is being reached around the present epoch. In this sense, the model is not able to solve the coincidence problem.

We have found that there is a negative correlation between the value of the age of the Universe today and the current equation of state of dark energy: larger values of $\omega_{\phi 0}^{(e)}$ correspond to a lower age of the Universe. This result is the opposite of the case in which the equation of state of dark energy is constant.

A generic feature of this class of models is that the Universe is older than noncoupled dark energy models. In fact, we found $t_{0}=15.3_{-0.7}^{+1.3} \mathrm{Gyr}$. Better model independent determination of the age of the Universe could help to distinguish among different contenders for explaining the origin of the dark energy.

\section{ACKNOWLEDGMENTS}

We would like to thank $\mathrm{Z}$. Chacko for asking the right question. This work was supported by Fundação de Amparo à Pesquisa do Estado de São Paulo (FAPESP), grant 01/ 11392-0, and by Conselho Nacional de Desenvolvimento Científico e Tecnológico (CNPq).
[1] D.N. Spergel et al., Astrophys. J., Suppl. 148, 175 (2003).

[2] P.J.E. Peebles and B. Ratra, Rev. Mod. Phys. 75, 559 (2003).

[3] M.S. Turner, Phys. Rep. 333, 619 (2000).

[4] R.R. Caldwell, R. Dave, and P.J. Steinhardt, Phys. Rev. Lett. 80, 1582 (1998).

[5] B. Ratra and P.J.E. Peebles, Phys. Rev. D 37, 3406 (1988); P.J.E. Peebles and B. Ratra, Astrophys. J. Lett. 325, L17 (1988).

[6] C. Wetterich, Nucl. Phys. B302, 668 (1988).

[7] For reviews, see, e.g., J. Ellis, Philos. Trans. R. Soc. London A361, 2607 (2003); K.A. Olive, astro-ph/0301505.

[8] G.W. Anderson and S.M. Carrol, astro-ph/9711288.

[9] J.A. Casas, J. García-Bellido, and M. Quirós, Class. Quantum Grav. 9, 1371 (1992).

[10] G.R. Farrar and P.J.E. Peebles, astro-ph/0307316.

[11] M.B. Hoffman, astro-ph/0307350.

[12] L. Amendola, Phys. Rev. D 62, 043511 (2000).
[13] L. Amendola and D. Tocchini-Valentini, Phys. Rev. D 64, 043509 (2001).

[14] L. Amendola, Mon. Not. R. Astron. Soc. 342, 221 (2003).

[15] M. Pietroni, Phys. Rev. D 67, 103523 (2003).

[16] D. Comelli, M. Pietroni, and A. Riotto, Phys. Lett. B 571, 115 (2003).

[17] E.J. Copeland, A.R. Liddle, and D. Wands, Phys. Rev. D 57, 4686 (1998).

[18] A.R. Liddle and R.J. Scherrer, Phys. Rev. D 59, 023509 (1998).

[19] U. França and R. Rosenfeld, J. High Energy Phys. 10, 015 (2002).

[20] W. Freedman et al., Astrophys. J. 553, 47 (2001).

[21] L.M. Krauss and B. Chaboyer, Science 299, 65 (2003).

[22] R. Jimenez, L. Verde, T. Treu, and D. Stern, Astrophys. J. 593, 629 (2003).

[23] L.M. Krauss, astro-ph/0212369.

[24] M. Kunz, P.S. Corasaniti, D. Parkinson, and E.J. Copeland, astro-ph/0307346. 